\title{
3 Research Square

\section{Hypercaloric diets impair ovarian follicular development by inducing hyperinsulinemia and hyperlipidemia in female mice}

\section{Qiaoli Zhang}

Capital Medical University

\section{Yan Wang}

Xi'an Jiaotong University School of Medicine

Jiansheng Liu

Shanghai Jiaotong University: Shanghai Jiao Tong University

\section{Zi-Jiang Chen}

Shandong University

Yanzhi Du ( $\nabla$ yanzhidu@hotmail.com )

Shandong University https://orcid.org/0000-0002-7322-3390

\section{Research}

Keywords: Hypercaloric diets, Hyperinsulinemia, Hyperlipidemia, Follicular development

Posted Date: September 25th, 2020

DOl: https://doi.org/10.21203/rs.3.rs-81417/v1

License: (9) This work is licensed under a Creative Commons Attribution 4.0 International License. Read Full License 


\section{Abstract}

Long-term hypercaloric diets adversely impact ovarian follicular development and fecundity. We investigated the effects of high sugar (HS), high fat low sugar (HFLS), and high fat normal sugar (HFNS) diets on ovarian follicular development by feeding mice for up to 180 days. Body weight, gonadal fat, glucose, lipid, insulin, estrous cycle, sex hormones, ovarian tissues, and follicle ultrastructure were examined, and the expression of metabolism-related proteins was evaluated immunoblotting in ovarians. The mice on hypercaloric diets showed hyperinsulinemia and hyperlipidemia and exhibited heavier body and gonadal fat weights, longer estrous cycles, and fewer numbers of preantral and antral follicles; and the follicles that did form had impaired organelle morphology. The sex hormone levels in blood were similar to controls, excepting significantly elevated estradiol levels for the HS diet. In ovarian tissues, AMPKa phosphorylation was reduced while AKT phosphorylation and caspase-3 were increased in ovarian tissues in mice on all three hypercaloric diets. The data from our study collectively indicates possible mechanisms through which long-term exposure to unhealthy hypercaloric diets may impair ovarian follicular development: hyperinsulinemia and hyperlipidemia.

\section{Introduction}

Fertility disorders constitute an increasingly common public health problem worldwide [1], and currently affect about $13-17 \%$ of couples of reproductive age [2]. The absolute number of infertile couples increased from 42.0 million in 1990 to 48.5 million in 2010 [3], and the lifetime prevalence of infertility is about $17-28 \%$ [4]. The growing phenomenon of infertility has prompted recognition of infertility as a social disease by the World Health Organization (WHO).

Genetic factors, advanced age, autoimmune conditions, environmental toxins, and exposure to chemotherapy or radiotherapy may cause perturbed follicular development abnormalities in primordial follicles and result in early depletion of the ovarian follicular reserve [5]. There is an upward trend in the incidence of idiopathic infertility, which may be associated with unhealthy diet and lifestyle may affect ovarian function, and unhealthy dietary patterns have been associated with anovulatory infertility [6]. Human fertility depends on nutrition; however, both malnourishment and overnourishment impair fertility. Hypercaloric diets can lead to development of obesity and metabolic dysfunction in humans and animals [7], and being underweight and being overweight can increase risk for infertility [8]. Obesity is recognized as a global epidemic by the $\mathrm{WHO}$ and it can lead to a wide range of secondary pathologies including female infertility [9].

Metabolic dysfunctions are often closely related to reproductive abnormalities [10], and dietary habits have been implicated in significant effects on ovarian development [11]. Blood circulating metabolites such as glucose, lipids, and hormones like insulin are known to affect the hypothalamic-pituitary-ovarian axis, and may induce infertility in women [12]. A study showed that diet-induced obese mice had significantly more apoptotic ovarian follicles, smaller and fewer mature oocytes, smaller fetuses, and smaller pups compared to normal mice [13]. Other studies have shown that diet-induced obesity affects 
oocyte meiotic maturation, ovulation, and fertilization [14]. Moreover, such diets can reduce oocyte quality and blastocyst survival rates, cause abnormal embryonic cellular differentiation, and increase the rates of early embryo loss and fetal growth retardation [15].

Liver kinase B1 (LKB1), also known as serine/threonine kinase 11 (STK11), is a serine/threonine protein kinase which has been implicated in a number of key cellular processes including the regulation of cell proliferation, cell polarity, and energy metabolism $[16,17]$. Shaw et al. demonstrated that LKB1 is a major upstream kinase responsible for activation of AMP-activated protein kinase (AMPK), which acts as a metabolic rheostat to maintain energy homeostasis in response to a decline in the cellular ATP/AMP ratio [18]. Other studies have also linked LKB1 to phosphorylation-mediated regulation of AMPK $[19,20]$ and to microtubule dynamics [21].

One of the major downstream signaling pathways controlled by LKB1/AMPK is the mammalian target-ofrapamycin (mTOR) pathway, which appears to be responsible for controlling protein synthesis, cell growth, and protecting against apoptosis during cellular stress [22]. It is known that AKT (also known as protein kinase $B$ ) is activated by serine and threonine phosphorylation and that AKT functions as a key mediator of insulin signaling [5]. AKT is involved in many cellular processes including cell growth, survival, proliferation, and metabolism [23]. Medically, AKT dysregulation has been associated with several human diseases including infertility and ovarian cancer [24].

Young women in reproductive age and even prepubertal girls often have consumed hypercaloric diets with excessive amounts of sugar or/and fat, which can contribute to hyperinsulinemia and hyperlipidemia. The effects of these unhealthy diets on female fecundity have not been thoroughly studied, and cellular mechanisms that function in energy balance for reproduction are not well understood. Here, we investigated the possible effects of different unhealthy diets on ovarian follicular development in prepubertal $\mathrm{C} 57 \mathrm{BL} / 6 \mathrm{~J}$ female mice to address the hypothesis that long-term consumption of hypercaloric diets may result in abnormalities of glucose and lipid metabolism and may eventually impair ovarian follicular development in female mice. We fed mice with three different hypercaloric diets, including high sugar (HS), high fat low sugar (HFLS), and high fat normal sugar (HFNS). We also studied the molecular mechanism(s) associated with the observed effects from the hypercaloric diets by investigating the signaling pathways which were altered by the diets, and found that dysfunction of such pathways (which influence for example cell proliferation, growth, survival, and energy metabolism) may deleteriously affect ovarian follicular development.

\section{Materials And Methods}

\section{Animal experiments}

C57BL/6J female mice (body weight 13-16 g, 28 days old) were obtained from the Laboratory Animal Center of Xi'an Jiaotong University (Xi'an, Shaanxi, China) and housed up to five mice per cage in a temperature-controlled room with a 12/12 $\mathrm{h}$ light/dark. The mice were fed with one of the diets detailed in 
Table 1 (HS, HFLS, HFNS, or standard Purina rodent chow) for up to 180 consecutive days; and the mice were weighed every two weeks. There were 4 groups with 30 mice for each group in the study. The mice chow was purchased from Beijing Keaoxieli feed Co. LTD (Beijing, China). The food and water were ad libitum to all animals. The animal experiments were approved by the Committee on the Use and Care of Animals of Xi'an Jiaotong University (Xi'an, Shaanxi, China; No. 2014-006) and all experimental procedures were carried out in accordance with approved animal protocols.

Table 1

The composition of chow nutrients for the normal (Control), HS, HFLS, and HFNS diets

\begin{tabular}{|c|c|c|c|c|c|c|c|c|}
\hline \multirow[t]{2}{*}{ Nutrients } & \multicolumn{2}{|c|}{ Control Diet } & \multicolumn{2}{|c|}{ HS Diet } & \multicolumn{2}{|c|}{ HFLS Diet } & \multicolumn{2}{|c|}{ HFNS Diet } \\
\hline & $\%$ & Kcal \% & $\%$ & Kcal \% & $\%$ & Kcal \% & $\%$ & Kcal \% \\
\hline Protein & 20.0 & 24.4 & 16.5 & 18.5 & 18.9 & 15.1 & 13.2 & 12.3 \\
\hline Fat & 4.5 & 12.3 & 3.8 & 9.5 & 33.5 & 59.9 & 19.1 & 39.8 \\
\hline Carbohydrate & 52.0 & 63.3 & 64.3 & 72.0 & 31.5 & 25.0 & 52.0 & 47.9 \\
\hline $\begin{array}{l}\text { Total calories } \\
\text { (Kcal/g) }\end{array}$ & - & 3.3 & - & 3.6 & - & 5.0 & - & 4.3 \\
\hline $\begin{array}{l}\text { Note. The left } \\
\text { in total becau } \\
\text { The right colu } \\
\text { dietary pattern } \\
\text { vitamin, miner }\end{array}$ & $\begin{array}{l}n \text { is t } \\
\text { er con } \\
\text { he pe } \\
\text { calor } \\
\text { It, su }\end{array}$ & $\begin{array}{l}\text { ercenta } \\
\text { sitions } \\
\text { itage o } \\
\text { are } 100 \\
\text { e, lard, }\end{array}$ & $\begin{array}{l}\text { of pro } \\
\text { h as r } \\
\text { lories } \\
\text { The s }\end{array}$ & $\begin{array}{l}\text {, fat, and } \\
\text { erals, vita } \\
\text { nsisting } \\
\text { ces of nu } \\
\text { fat, and }\end{array}$ & $\begin{array}{l}\text { bohyc } \\
\text { and } \\
\text { otein, } \\
\text { tts inc } \\
\text { estero }\end{array}$ & $\begin{array}{l}\text { te in each } \\
t \text { are not } \\
\text { t, and car } \\
\text { de corn, } t\end{array}$ & $\begin{array}{l}\text { t and } \\
\text { uded it } \\
\text { ydrate } \\
\text { bean }\end{array}$ & $\begin{array}{l}\text { not } 100 \% \\
\text { he Table. } \\
\text { each } \\
\text { ke, }\end{array}$ \\
\hline
\end{tabular}

\section{Sample collection}

One week before the mice were fed their particular diets for 180 days, venous blood samples were collected using glass capillaries from tails; these samples were used for monitoring of serum lipids (after mice were fasted for $12 \mathrm{~h}$ ). At the end of the experiment (day 180), all mice were weighed and anesthetized with pentobarbital $(150 \mathrm{mg} / \mathrm{kg})$ by intraperitoneal injection. Blood samples were collected by cardiac puncture from the mice after fasting for $12 \mathrm{~h}$, and placed in tapered plastic centrifuge tubes. The blood samples were centrifuged at $1,500 \times \mathrm{g}$ for $15 \mathrm{~min}$, and the serum was isolated and stored at $-80^{\circ} \mathrm{C}$ for assays of sex hormones, insulin. Tissues including ovaries and gonadal fats were collected. The freshly dissected ovaries were snap-frozen in liquid nitrogen for further analysis of protein lysates or were immediately fixed in $4 \%$ paraformaldehyde for histopathology. The right ovary was used for histological studies and the left ovary for molecular studies.

\section{Blood sample assay}

Blood lipids, including total cholesterol (TC), triglycerides (TG), high-density lipoprotein cholesterol (HDLC) and low-density lipoprotein cholesterol (LDL-C) were analyzed using commercial kits (Biosino). The day sacrificing the mice, the fasting blood glucose (FBG) was measured by glucometer with glucose strips. The estradiol $\left(E_{2}\right)$, fasting blood insulin $(F B I)$, and testosterone $(T)$ were detected by enzyme-linked 
immunosorbent assay (ELISA) kits from ALPCO after the mice were on different diets for 180 days. A homeostasis model assessment index of insulin resistance (HOMA-IR) value was calculated using the formula: blood glucose $(\mathrm{mmol} / \mathrm{L}) \times$ serum insulin $(\mathrm{mIU} / \mathrm{L})] / 22.5$. There were 20 mice used for each experimental group.

\section{Estrous cycle}

To study the effect of hypercaloric diets on estrous cycle, vaginal smears were taken with a homemade miniature cotton swab and cytologically evaluated microscopically via cellular staining with hematoxylin and eosin (H\&E) after the mice were on the various diets for 180 days. The vaginal smears were obtained starting at 15 days before the end of the experiment for 15 consecutive days (on day 166-180). There were 10 mice used for each experimental group.

\section{Ovarian section}

The ovaries were fixed in $4 \%$ paraformaldehyde for $24 \mathrm{~h}$, and dehydrated in increasing concentrations of ethanol, followed by immersion in xylene, and then embedding in paraffin wax. The ovary samples were serially sectioned at $5 \mu \mathrm{m}$ using a Leica microtome (Leica RM 2235). The paraffin sections were deparaffinized in xylene twice and then rehydrated in decreasing concentrations of ethanol. The ovarian sections were then stained with H\&E and analyzed under a conventional light microscope. The section was randomly selected from amongst serial sections (starting after fifth section) of ovary samples for the follicle count. There were 6 mice used for each experimental group.

\section{Follicle count}

The numbers of primordial, primary, preantral, and antral follicles were counted in one section from every fifth ovarian section. All stages of follicles, including atresia follicles, were counted in the study. The counting was blindly performed by a single person to avoid the variation caused by different individuals and only the follicles containing an oocyte with a visible nucleolus were counted to avoid counting the same follicle twice. The numbers of the follicles in the selected sections were then multiplied by 5 to estimate of the total number of follicles in each ovary. The classification and computing methods of the follicles were used from the method reported previously $[25,26]$. There were 10 mice used for each experimental group.

\section{Transmission electron microscopy observation of ultrastructure of follicles}

Ovaries were cut into $1 \mathrm{~mm}^{3}$ sections on ice and fixed in $2.5 \%$ glutaraldehyde in $0.1 \mathrm{M}$ phosphate buffer. Thereafter, the ovarian tissues were fixed in osmium tetroxide, dehydrated, and embedded in epoxy resin. Ultra-thin sections of the samples were stained with lead citrate and uranyl acetate and subsequently analyzed with a transmission electron microscope (HITACHI, $\mathrm{H}-7650)$ to observe the ultrastructures of follicles. There were 6 mice used for each experimental group.

\section{Western blotting}


Samples of ovarian protein extracts were mixed with sodium dodecyl sulfate sample buffer and boiled for $8 \mathrm{~min}$. Equal amounts of proteins were then loaded into sodium dodecyl sulfate-polyacrylamide gel for electrophoresis and subsequently transferred onto polyvinylidene fluoride membranes. The membranes were blocked in $5 \%$ nonfat milk for $2 \mathrm{~h}$ at room temperature and incubated with primary antibodies with an appropriate dilution overnight at $4{ }^{\circ} \mathrm{C}$, followed by incubation for $60 \mathrm{~min}$ at room temperature with secondary antibodies at a dilution of 1:3,000 (anti-rabbit GGHL-15p and anti-mouse GGHL-90p). Chemiluminescence reagent (Thermo, OH189776, Thermo Fisher Scientific Inc, Wilmington, $D E$, USA) was used to visualize the blots. Densitometric analysis of protein levels of ovaries from the experimental and control mice was then conducted. All western blotting analyses were repeated at least three times, with 6 mice for each experimental group. The antibody information is shown in Supplementary Table 1.

\section{Statistical analysis}

All data are presented as means \pm standard errors (SE). Statistical analyses were performed using SPSS 19.0 statistical software. All data were compared using one-way analysis of variance (ANOVA) followed by the Dunnett-t test. A $p<0.05$ was considered to be statistically significant.

\section{Results}

\section{Changes of the body and gonadal fat weights in mice on hypercaloric diets}

The body weights of mice did not differ between the control and experimental groups in the pretreatment phase $(p>0.05)$. In spite of weight fluctuations, at the end of the experiment (180 days), the body weights of all mice on hypercaloric diets were significantly higher than the control mice $(p<0.05)$; the mice on the HFNS diet had the most substantial increase in body weight (Fig. 1A). The weights of gonadal fat pads and the ratios of gonadal fat pad to body weight in all mice on hypercaloric diets were significantly higher than the control mice $(p<0.05, p<0.01$ or $p<0.001)$ (Fig. 1B \& C).

\section{Lipid and glucose metabolic abnormalities in the mice on hypercaloric diets}

After180 days on diets, there was a significant increase the levels of TG, TC, and HDL-C in all mice on hypercaloric diets compared to the control mice $(p<0.05, p<0.01$ or $p<0.001)$. However, the LDL-C levels did not differ significantly $(p>0.05)$ only in the mice with HFNS diet compared to the control mice (Fig. 2A). The FBG levels were similar $(p>0.05)$ between the mice on hypercaloric diets and the control mice. The FBI levels were significantly increased in all mice on hypercaloric diets compared to control mice $(p<0.05)$, and the HOMA-IR were significantly elevated in all mice on hypercaloric diets $(p<0.05$ or $p<0.01$ ) with mice on the HS diet exhibiting the highest HOMA-IR values ( Fig. 2B). 


\section{Sex hormone levels and estrous cycle in mice on hypercaloric diets}

There were no significant changes $(p>0.05)$ in serum concentrations of $T$ in the mice fed the HS, HFLS, or HFNS diets for 180 days. However, the $\mathrm{E}_{2}$ concentration was significantly increased in the mice on the HS diet as compared to the control mice $(p<0.05$, Fig. $3 A)$.

The mice on the HS, HFLS, and HFNS diets for 180 days had extended estrous cycles and prolonged proestrus, metestrus, and diestrus phases as compared to control mice $(p<0.05, p<0.01$ or $p<0.001$, respectively), but the estrus phase was similar ( $p>0.05)$ between all of the mice (Fig. 3B).

\section{Follicle count and follicle ultrastructure in mice on hypercaloric diets}

Macroscopic observation of ovaries revealed that the control mice exhibited morphologically normal follicles and normal corpora lutea but that the numbers of follicles were significantly decreased in the ovaries of the mice fed each of the three hypercaloric diets (Fig. 4). Moreover, the numbers of preantral and antral follicles were significantly decreased in all experimental mice compared to the control mice ( $p$ $<0.05)$ (Fig. 5).

In control mice, the follicles had abundant cytoplasmic organelles, including bacilliform mitochondria, smooth endoplasmic reticulum, ribosomes, and Golgi complexes in the oocytes at various stages, and had nuclei with rich and evenly distributed chromatin. The follicle microstructures in the mice fed the HS, HFLS, and HFNS diets showed impairment of follicles, with irregular mitochondria, Golgi, and zona pellucida, irregular granulosa cell arrangement, and apparent apoptosis; further their microvilli on the luminal surface of the oocyte and granulosa cells were rare or completely absent (Fig. 6).

Effects of p-AMPK ${ }_{a}$ reduction and p-AKT and caspase- 3 enhancements in the ovaries of mice fed hypercaloric diets

There were no significant differences $(p>0.05)$ in $p$-LKB1 and p-mTOR levels, whereas p-AMPK ${ }_{a}$ was significantly decreased $(p<0.05)$ in the ovaries of the mice on HS, HFLS, and HFNS diet compared to the control mice (Fig. 7). Nor did we observe significant differences $(p>0.05)$ in the levels of P85 and P110. However, the p-AKT and caspase-3 levels were significantly increased $(p<0.05)$ in the ovaries of the mice fed the HS, HFLS, and HFNS diets compared to control mice (Fig. 8).

\section{Discussion}

We studied the effect of different hypercaloric diets on ovarian follicular development at both the tissue and molecular levels in a mouse model. We found that the mice on hypercaloric diets for 180 days developed hyperinsulinemia and hyperlipidemia, with significantly increased body and gonadal fat weights. At the cellular level, hypercaloric diets induced the impairment of follicle organelles, reduced the 
numbers of preantral and antral follicles, and prolonged estrous cycles. We also observed disequilibrium in energy metabolism in the ovarian tissues of mice fed the hypercaloric diets, as evidenced by significantly decreased AMPK activation but increased AKT activation in ovaries. Collectively, our data hint at possible mechanisms which may underlie the known negative impacts of obesity on female fecundity, including a role for hyperinsulinemia and/or hyperlipidemia on ovarian follicular development and possibly the pathogenesis of folliculogenesis disorders.

Extensive previous work has established that female fertility can be affected by obesity at all stages of reproduction including oocyte development $[12,15,27-29]$. We were particularly interested in the impact of unhealthy diets on ovarian follicular development, and fed female mice with hypercaloric diets that provide high caloric content with different ratios of sugar and fat (and very slight differences in protein levels) to mimic a variety of unhealthy human diets. The total body weights of all mice on hypercaloric diets were significantly increased compared to controls after 180 days.

Examination of specific tissues after sacrificing the mice revealed that the gonadal fat weights of the hypercaloric-diet-fed mice were also significantly increased compared to the control. There was an obvious trend for weight gain to these fat deposits near the reproductive tissue: the hypercaloric diets with relatively higher fat content led to more substantial weight gains. Fat deposits have been reported to produce lipotoxic effects on female fertility [30]. Analyses of blood samples in our study revealed that each of the hypercaloric diets significantly increased blood lipids (e.g., TG, TC, and HDL-C). Moreover, considering that both overabundant lipids and insulin in blood circulation have been shown to cause unfavorable reproductive environment-hyperinsulinemia causes early responses to luteinizing hormones on granulosa cells of small follicles and causes anovulation because of premature differentiation of these cells [31]. It was highly notable that we also observed hyperinsulinemia in the mice fed all of the hypercaloric diets.

In terms of reproductive phenotypes, we found that the mice fed hypercaloric diets had significantly longer estrous cycles, with prolonged proestrus, metestrus, and diestrus phases as compared to control mice, suggesting the potential for affected reproductive capacity. The aforementioned metabolic disturbances were associated with delayed estrous cycles and with hindered development of oocytes and granulosa cells, results consistent with the findings of Minge [15].

Ovaries are known to as target organs for insulin action; they exhibit insulin receptors in both their stromal and follicular compartments, and insulin has been shown to stimulate steroidogenesis in theca cells and granulosa cells in vitro and is involved in follicular development and granulosa cell proliferation [32]. Granulosa cells provide nutritional support for the oocyte, regulate oocyte growth, and maintain meiotic arrest, and hyperinsulinemia can affect follicle development and ovulation [33]. Our results indicate that long-term hypercaloric dietary exposure can interfere with follicular growth, specifically by reducing the numbers of preantral and antral follicles and by inhibiting the growth of the primary follicle.

Further, in light of work of previous about reproductive disorders in mice has shown that high fat diets can negatively affect folliculogenesis, fertilization, and oocyte quality [34, 35], and given that hypercaloric 
diets have been shown to cause mitochondrial abnormalities and meiotic defects [36], we speculate that the abnormal follicles, disrupted oocytes and granulosa cells, and abnormal morphology of mitochondria, Golgi, and zona pellucida we observed hint at a mechanism wherein ultrastructural follicle defects may reduce the fecundity of female mice fed hypercaloric diets. Specifically, compared to the normal diet, we found that for the HS, HFLS, and HFNS mice, the granulosa cells were rare and exhibited endoplasmic reticulum dilatation, which presented characteristic morphological changes of apoptosis. The boundary of the zona pellucida is unclear: the zona pellucid had different thicknesses and the electron density was uneven, and the oocyte microvillis were substantially reduced.

Female fertility is highly dependent on the regulation of energy metabolism. AMPK is an important cellular energy sensor that is activated in response to ATP deficits and acts by shutting down energy consumption and turning on energy-generating pathways [37]. The potential involvement of AMPK in reproduction and more specifically in ovarian follicle development has been reported [11]. Our study found that the activation of p-AMPKa was reduced, while AKT phosphorylation and caspase-3 were increased in the ovaries of female mice on the three hypercaloric diets, suggesting the interesting idea that the ovaries of mice fed hypercaloric diets exhibit increased apoptosis. It may be possible to further this initial insight about an association between apoptosis and excessive energy intake by using highly spatially resolved sampling from mice or perhaps by manipulating the micronutrient sources in culture media during in vitro work.

\section{Conclusions}

We found that AKT phosphorylation was increased in mice with long-term hypercaloric diets and also noted increased caspase-3 accumulation. Although much remains to be clarified about the effects of both high sugar and high fat diets on the cellular energy metabolism and programmed death in ovaries, our study provides preliminary evidence to support the speculation that the hyperinsulinemia and hyperlipidemia that result from hypercaloric diets may deleteriously effect female fecundity by causing a dysregulation of folliculogenesis. Our study may help to develop potential preventative measures and interventions for women with unhealthy diets seeking improved reproductive follicular development, a problem with major have clinical and social significance.

\section{Declarations}

\section{Availability of data and materials}

All data generated or analysed during this study are included in this published article and its supplementary information files.

\section{Ethics approval and consent to participate}


The animal experiments were approved by the Committee on the Use and Care of Animals of Xi'an Jiaotong University (Xi'an, Shaanxi, China; No. 2014-006).

\section{Consent for publication}

Not applicable.

\section{Competing interests}

The authors declare that they have no competing interests.

\section{Funding}

This study was supported by the National Natural Sciences Foundation of China (81490743 and 81302441), and the China Postdoctoral Science Foundation funded project (2014M561488).

\section{Authors' contributions}

Yanzhi Du and Zi-Jiang Chen conceived and designed the experiments; Qiaoli Zhang, Yan Wang and Jiansheng Liu performed the experiments; Qiaoli Zhang analyzed the data and wrote the manuscript.

\section{Acknowledgements}

We are indebted to Professor Enqi Liu from Laboratory Animal Center, School of Medicine, Xi'an Jiaotong University. We thank Dr. Weitao Hu, the Second Affiliated Hospital of Xi'an Jiaotong University with the collection of blood and ovarian samples and Professor Yaling Zhao for help with the statistics. We also thank Dr. Shousong Cao for critical reviewing, proof-reading, and editing the manuscript.

\section{References}

1. Boivin J, Bunting L, Collins JA, \& Nygren KG. International estimates of infertility prevalenceand treatment-seeking: potential need and demand for infertility medical care. Hum Reprod. 2007;22:1506-1512.

2. Sińska B, Kucharska A, \& Dmoch-Gajzlerska E. The diet in improving fertility in women. Pol. Merkur Lekarski. 2014;36:400-402.

3. Mascarenhas MN, Flaxman SR, Boerma T, Vanderpoel S, \& Stevens GA. National, regional, and global trends in infertility prevalence since 1990: a systematic analysis of 277 health surveys. PLoS Med. 2012;9:e1001356.

4. Schmidt L. Social and psychological consequences of infertility and assisted reproduction-what are the research priorities? Hum Fertil (Camb). 2009;12:14-20. 
5. Mackenzie RW, \& Elliott BT. Akt/PKB activation and insulin signaling. a novel insulin signaling pathway in the treatment of type 2 diabetes. Diabetes. Metab Syndr Obes. 2014;7:55-64.

6. Chavarro JE, Rich-Edwards JW, Rosner BA, \& Willett WC. Diet and lifestyle in the prevention of ovulatory disorder infertility. Obstet Gynecol. 2007;110:1050-1058.

7. Dhingra R, Sullivan L, Jacques PF, Wang TJ, Fox CS, Meigs JB, et al. Soft drink consumption and risk of developing cardiometabolic risk factors and the metabolic syndrome in middle-aged adults in the community. Circulation. 2007; 116:480-488.

8. Metwally $M$, Ong KJ, Ledger WL, \& Li TC. Does high body mass index increase the risk of miscarriage after spontaneous and assisted conception? A meta-analysis of the evidence. Fertil Steril. 2008;90:714-726.

9. Veleva Z, Tiitinen A, Vilska S, Hyden-Granskog C, Tomas C, Martikainen H, Tapanainen JS. High and Iow BMI increase the risk of miscarriage after IVF/ICSI and FET. Hum. Reprod. 2008;23:878-884.

10. Agarwal AK, Tunison K, Dalal JS, Nagamma SS, Hamra FK, Sankella S, Shao X, Auchus RJ, Garg A. Metabolic, Reproductive, and Neurologic Abnormalities in Agpat1-Null Mice. Endocrinology. 2017; 158:3954-3973.

11. Dupont J, Reverchon M, Cloix L, Froment P, \& Ramé C. Involvement of adipokines, AMPK, PI3K and the PPAR signaling pathways in ovarian follicle development and cancer. Int J Dev Biol. 2012:56:959-967.

12. Brothers KJ, Wu S, DiVall SA, Messmer MR, Kahn CR, Miller RS, et al. Rescue of obesity-induced infertility in female mice due to a pituitary-specific knockout of the insulin receptor. Cell Metab. 2010;12:295-305.

13. Jungheim ES, Schoeller EL, Marquard KL, Louden ED, Schaffer JE, \& Moley KH. Diet-induced obesity model: abnormal oocytes and persistent growth abnormalities in the offspring. Endocrinology. 2010;151:4039-4046.

14. Luzzo KM, Wang Q, Purcell SH, Chi M, Jimenez PT, Grindler N, et al. High fat diet induced developmental defects in the mouse: oocyte meiotic aneuploidy and fetal growth retardation/brain defects. PLoS One. 2012; 7:e49217.

15. Minge CE, Bennett BD, Norman RJ, \& Robker RL. Peroxisome proliferator-activated receptor-gamma agonist rosiglitazone reverses the adverse effects of diet-induced obesity on oocyte quality. Endocrinology. 2008; 149:2646-2656.

16. Hardie DG. The AMP-activated protein kinase pathway-new players upstream and downstream. J Cell Sci. 2004;117:5479-5487.

17. Alessi DR, Sakamoto K, \& Bayascas JR. LKB1-dependent signaling pathways. Annu Rev Biochem. 2006;75:137-163.

18. Shaw RJ, Kosmatka M, Bardeesy N, Hurley RL, Witters $L A$, DePinho RA, et al. The tumor suppressor $\angle K B 1$ kinase directly activates AMP-activated kinase and regulates apoptosis in response to energy stress. Proc Natl Acad Sci USA. 2004;101:3329-3335. 
19. Lizcano JM, Goransson O, Toth R, Deak M, Morrice NA, Boudeau J, et al. LKB1 is a master kinase that activates 13 kinases of the AMPK subfamily, including MARK/PAR-1. Embo J. 2004;23:833-843.

20. Jaleel M, McBride A, Lizcano JM, Deak M, Toth R, Morrice NA, et al. Identification of the sucrose nonfermenting related kinase SNRK, as a novel LKB1 substrate. FEBS Lett. 2005;579:1417-1423.

21. Kojima Y, Miyoshi H, Clevers HC, Oshima M, Aoki M, \& Taketo MM. Suppression of tubulin polymerization by the LKB1-microtubule-associated protein/ microtubule affinity-regulating kinase signaling. J Biol Chem. 2007;282:23532-23540.

22. Green AS, Chapuis N, Lacombe C, Mayeux P, Bouscary D, \& Tamburini J. KB1/AMPK/mTOR signaling pathway in hematological malignancies: from metabolism to cancer cell biology. Cell Cycle. 2017;10:2115-2120.

23. Los M, Maddika S, Erb B, \& Schulze-Osthoff $K$. Switching Akt. from survival signaling to deadly response. Bioessays. 2009;31:492-495.

24. Cecconi S, Mauro A, Cellini V, \& Patacchiola F. The role of Akt signaling in the mammalian ovary. Int J Dev Biol. 2012;56:809-817.

25. Hirshfield AN, \& Midgley AR Jr. Morphometri analysis of follicular development in the rat. Biol Reprod. 1978; 19:597-605.

26. Morita Y, Perez GI, Maravei DV, Tilly KI, \& Tilly JL. Targeted expression of Bcl-2 in mouse oocytes inhibits ovarian follicle atresia and prevents spontaneous and chemotherapy-induced oocyte apoptosis in vitro. Mol Endocrinol. 1999;13:841-850.

27. Inoki K, Zhu T, \& Guan KL. TSC2 mediates cellular energy response to control cell growth and survival. Cell. 2003;115:577-590.

28. Jungheim ES, Lanzendorf SE, Odem RR, Moley KH, Chang AS, \& Ratts VS. Morbid obesity is associated with lower clinical pregnancy rates after in vitro fertilization in women with polycystic ovary syndrome. Fertil Steril. 2009;92:256-261.

29. Brewer CJ, \& Balen AH. The adverse effects of obesity on conception and implantation. Reproduction. 2010;140:347-364.

30. Igosheva N, Abramov AY, Poston L, Eckert JJ, Fleming TP, Duchen MR, et al. Maternal diet-induced obesity alters mitochondrial activity and redox status in mouse oocytes and zygotes. PLoS One. 2010,5:e10074.

31. Willis DS, Watson $H$, Mason HD, Galea R, Brincat M, Franks S. Premature response to luteinizing hormone of granulosa cells from anovulatory women with polycystic ovary syndrome: relevance to mechanism of anovulation. J Clin Endocrinol Metab. 1998;83:3984-91.

32. Mukherjee S, \& Maitra A. Molecular \& genetic factors contributing to insulin resistance in polycystic ovary syndrome. Indian J Med Res.2010;131:743-760.

33. Diamanti-Kandarakis E, Argyrakopoulou G, Economou F, Kandaraki E, \& Koutsilieris M. Defects in insulin signaling pathways in ovarian steroidogenesis and other tissues in polycystic ovary syndrome (PCOS). J Steroid Biochem. 2008; 109:242-246. 
34. Sohrabi M, Roushandeh AM, Alizadeh Z, Vahidinia A, Vahabian M, \& Hosseini M. Effect of a high fat diet on ovary morphology, in vitro development, in vitro fertilisation rate and oocyte quality in mice. Singapore Med J.2015; 56:573-579.

35. Skaznik-Wikiel ME, Swindle DC, Allshouse AA, Polotsky AJ, \& McManaman JL. Subfertility and compromised ovarian function independent of obesity in mice. Biol Reprod.2016;94:108.

36. Seli E, Babayev E, Collins SC, Nemeth G, \& Horvath TL. Minireview. metabolism of female reproduction: regulatory mechanisms and clinical implications. Mol Endocrinol. 2014;28: 790-804.

37. Hardie DG. Minireview. the AMP-activated protein kinase cascade: the key sensor of cellular energy status. Endocrinology. 2003;144:5179-5183.

\section{Figures}
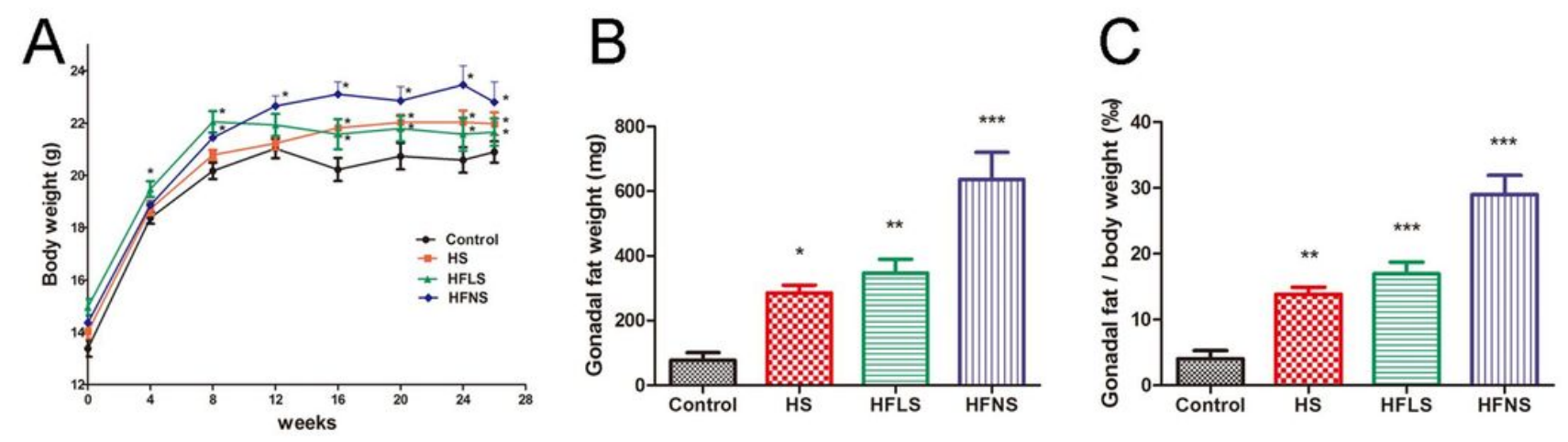

\section{Figure 1}

Body weight change curves and the weight of gonadal fat pads in mice on normal (control) or hypercaloric diets. (A) Body weight change curves in mice on normal (control), HS, HFLS, or HFNS diets; (B) The weight of gonadal fat pads; (C) The ratio of gonadal fat pad weight to body weight. The data are represented as the mean \pm SE from 30 different mice per experimental group $(n=30)$. C57BL/6J female mice were 28 days of age at $13-16 \mathrm{~g}$ body weight at the start of the various diet treatments. ${ }^{*} p<0.05 \mathrm{vs}$ Control. 

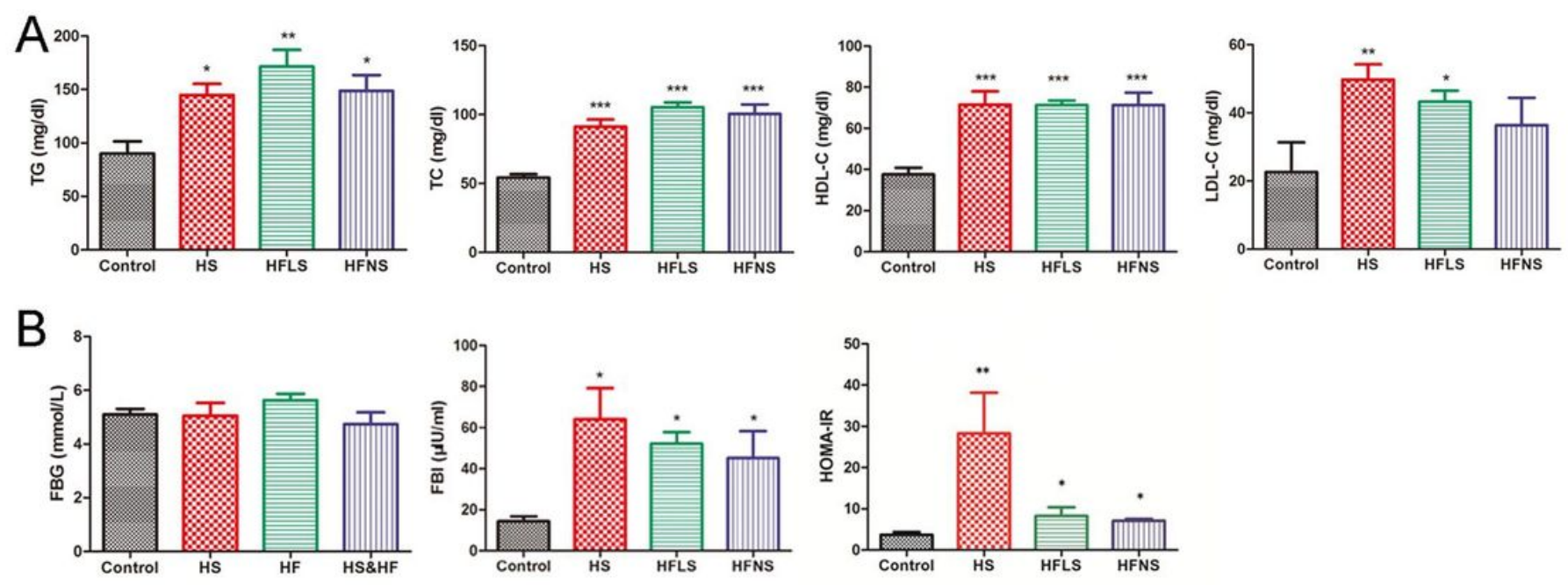

\section{Figure 2}

Summary of lipid and glucose metabolic parameters in mice on normal (control) or hypercaloric diets for 180 days. (A) The levels of serum TG, TC, HDL-C, and LDL-C; (B) The levels of FBG, FBI, and HOMA-IR. The data are represented as the mean \pm SE from 20 different mice per experimental group $(n=20) .{ }^{*} p<$ $0.05,{ }^{*} \mathrm{p}<0.01$, and ${ }^{* \star *} \mathrm{p}<0.001$ vs Control. 

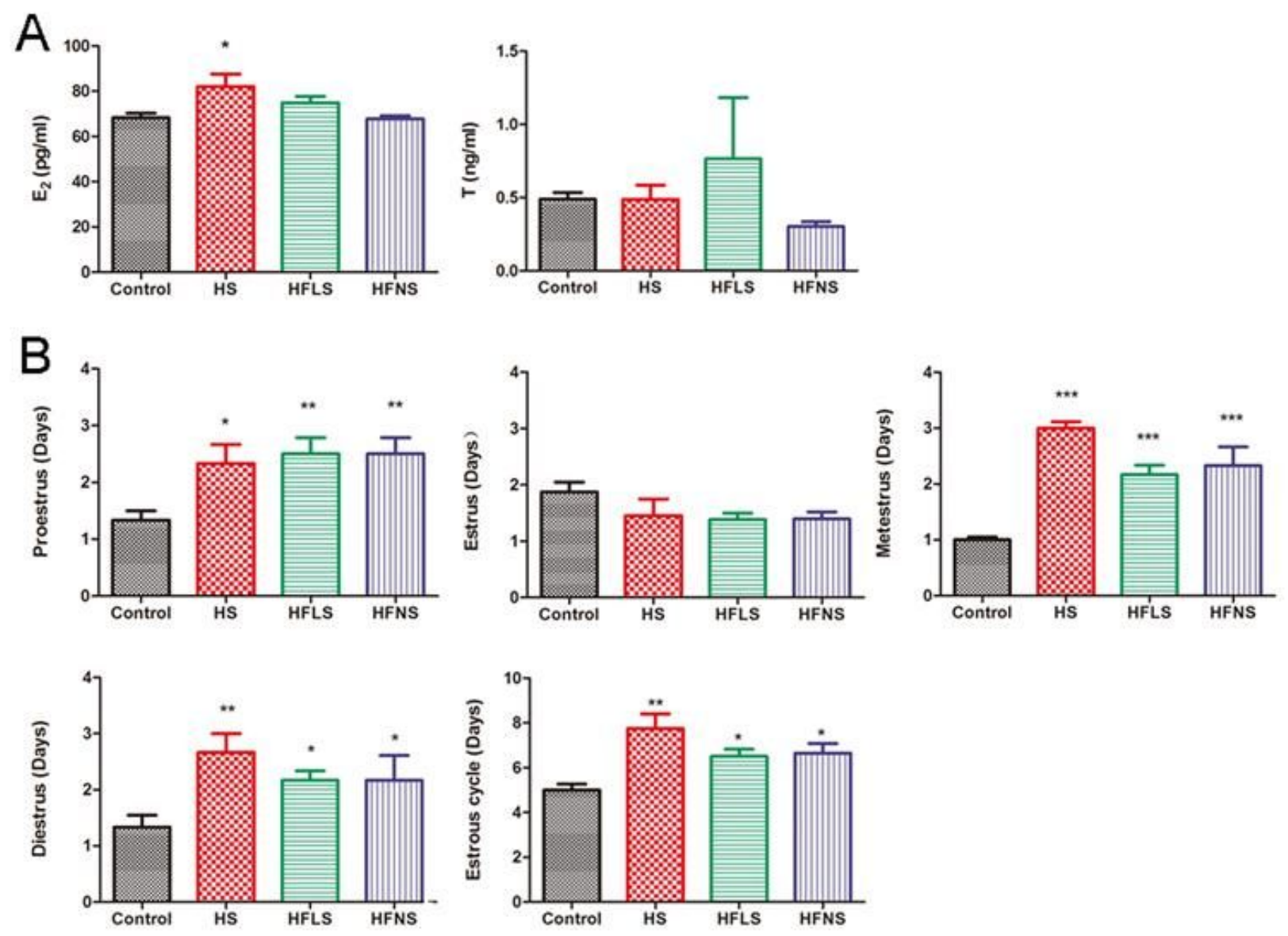

\section{Figure 3}

The concentrations of serum sex hormones in mice on control (control) or hypercaloric diets. (A) The serum concentrations of E2 and T from 20 different mice per experimental group $(n=20)$; (B) Proestrus, estrus, metestrus, diestrus phases and estrous cycle from 10 different mice per experimental group $(\mathrm{n}=$ 10). The data are presented as the mean \pm SE. ${ }^{\star} p<0.05,{ }^{*} p<0.01$, and ${ }^{* \star *} p<0.001$ vs Control. 


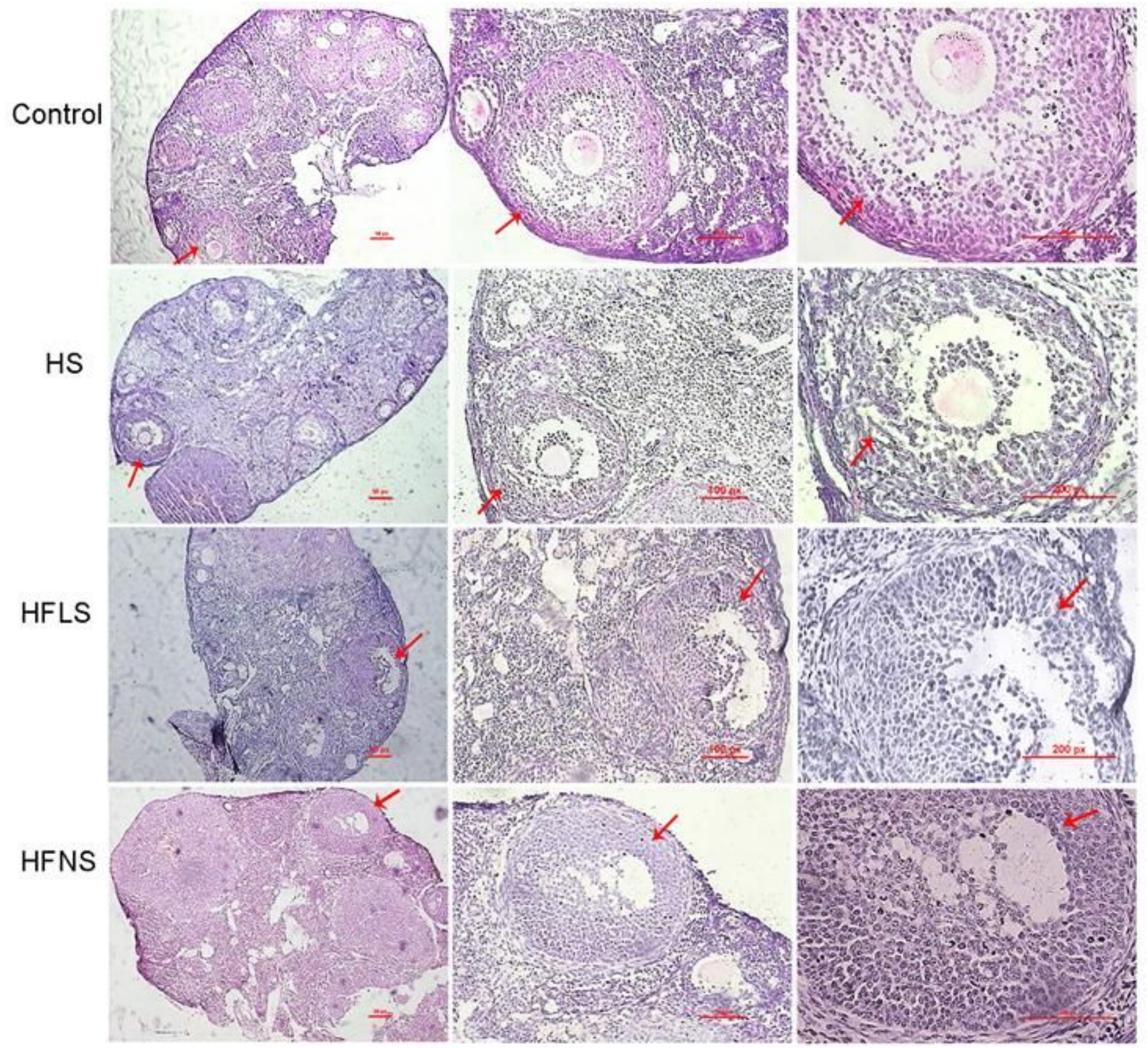

\section{Figure 4}

Morphological changes in the ovaries of mice fed normal (control), HS, HFLS, or HFNS diet for 180 days, assessed by hematoxylin and eosin (H\&E) staining. The representative ovary from the normal mouse shows follicles at different stages, including primordial follicles, primary follicles, preantral follicles, and antral follicles, and corpora lutea in the cortical ovarian zone, while there were obvious abnormal morphologies and a decrease in the number of follicles in the mice fed the HS, HFLS, or HFNS diets. Arrow: indicates the same follicle in each group. Six mice were used for each group $(n=6)$. 

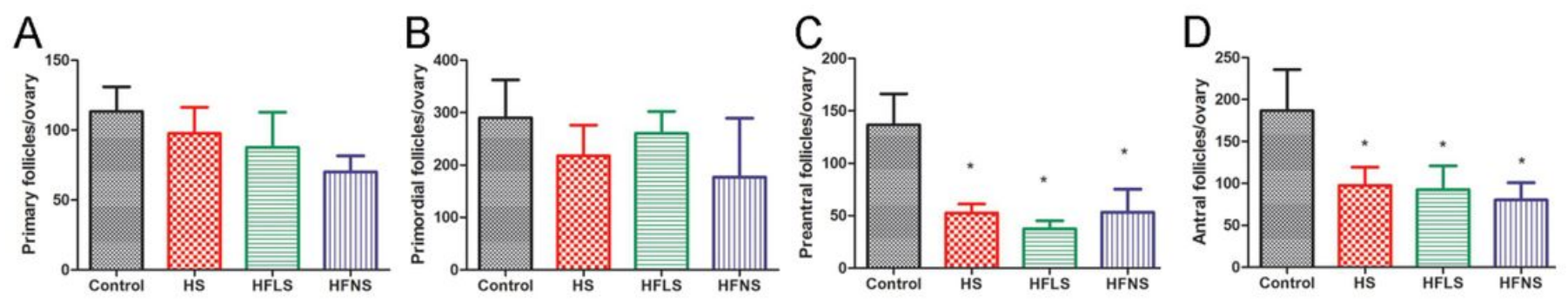

Figure 5

The numbers of follicles at different stages in mice on normal (control) or hypercaloric diets. (A) Number of primordial follicles per ovary; (B) number of primary follicles per ovary; (C) number of preantral follicles per ovary; (D) number of antral follicles per ovary. The data are presented as the mean \pm SE from six different mice per experimental group $(n=10) .{ }^{*} p<0.05$ vs Control. 


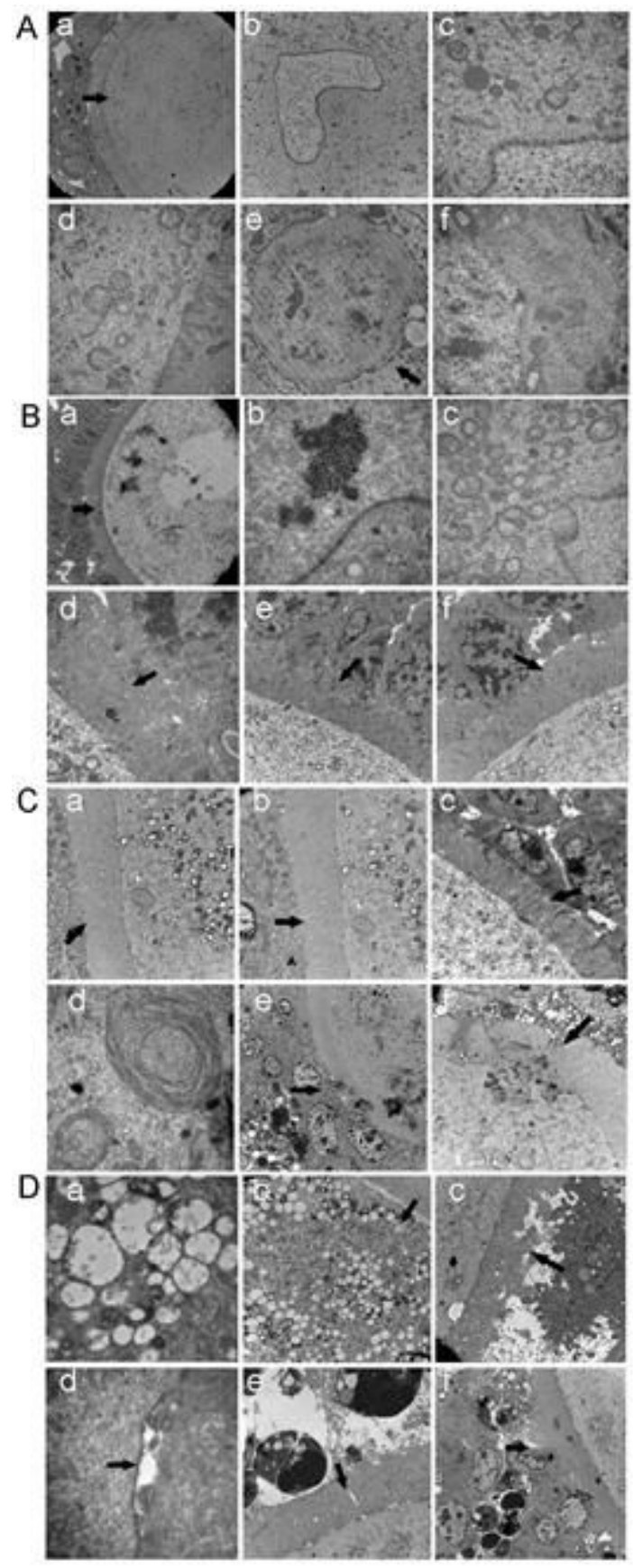

\section{Figure 6}

The morphologies of representative ovaries in mice on normal (control) or hypercaloric diets observed via transmission electron microscopy. (A) The representative control mouse. a: Oocyte with normal zona pellucida and granulosa cells ; b: Oocyte nucleus with irregular shape and around with cortical granula; c: The normal nuclear membrane with nuclear pore; $d$ : The normal ribosome and microvilli; e: The nucleus of an immature oocyte; $f$ : The normal structure of mitochondria. (B) epresentative HS diet mouse. a: Chromatic agglutination; b: nucleolus dissociation in the nucleus; $\mathrm{c}$ : mitochondria exhibited hyperplasia and edema; $\mathrm{d}$ : The microvilli on the luminal surface of the oocyte and granulosa cells were rare or not detectable; e: the boundary of the zona pellucida is unclear; $\mathrm{f}$ : zona pellucid of different thicknesses and electron densities. (C) Representative HFLS diet mouse. a: Mitochondria exhibited compensatory 
hypertrophy; b: A abundant lipid droplets in the cytoplasm; c: The oocyte microvilli were reduced; d: Golgi apparatus exhibited excessive bending and the occurrence of autophagy was obvious; e: granulosa cells apoptosis and apoptotic body formation; f: granulosa cells swelled into zona pellucida. (D)

Representative HFNS diet mouse. a: The swelling of mitochondria and cristae partially disappeared, with altered vacuoles; b: lipid droplets with a scattered distribution in oocytes; $c$ : the electron density of oocytes zona pellucida was sparse and uneven; $\mathrm{d}$ : there is a wide gap between oocytes and zona pellucida; e: the granulosa cells were endoplasmic reticulum dilatation and presented morphological changes characteristic of apoptosis; f: granulosa cells swelled into the partially dissolved zona pellucida. Arrow: indicating oocyte. There were six mice used for each group $(n=6)$.

A

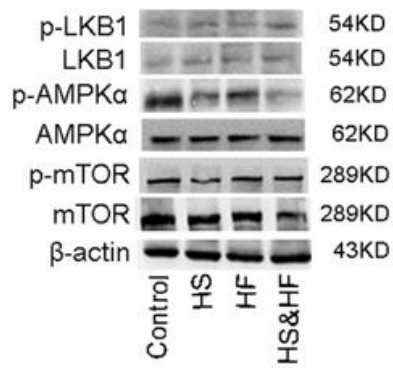

B

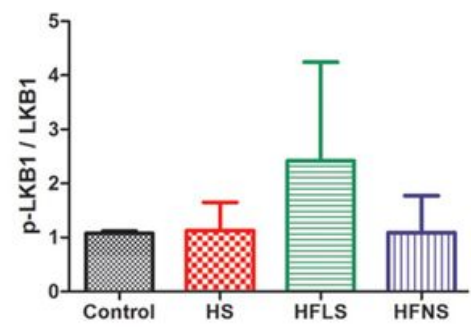

C

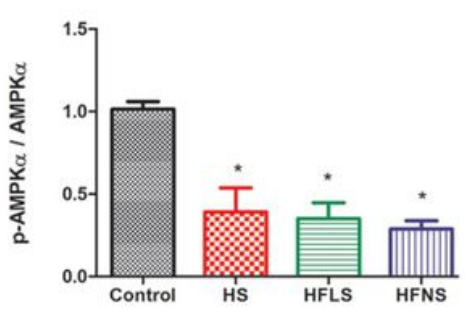

D

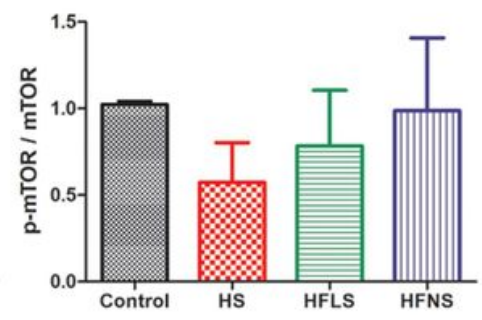

\section{Figure 7}

Effects of hypercaloric diets on the protein expression of AMPK/mTOR signal pathway in the ovarian tissues in mice on normal (control) or hypercaloric diets. (A) Western blotting gels of $p$-LKB1, LKB1, pAMPK囚, AMPK囚, p-mTOR, mTOR, and $\beta$-actin; (B) p-LKB1/LKB1; (C) p-AMPK囚/AMPK囚; and (D) p$\mathrm{mTOR} / \mathrm{mTOR}$. $\beta$-actin was used as a loading control. The data are presented as the mean $\pm \mathrm{SE}$ from six different mice per each experimental group $(n=6)$. ${ }^{*} p<0.05$ vs Control.

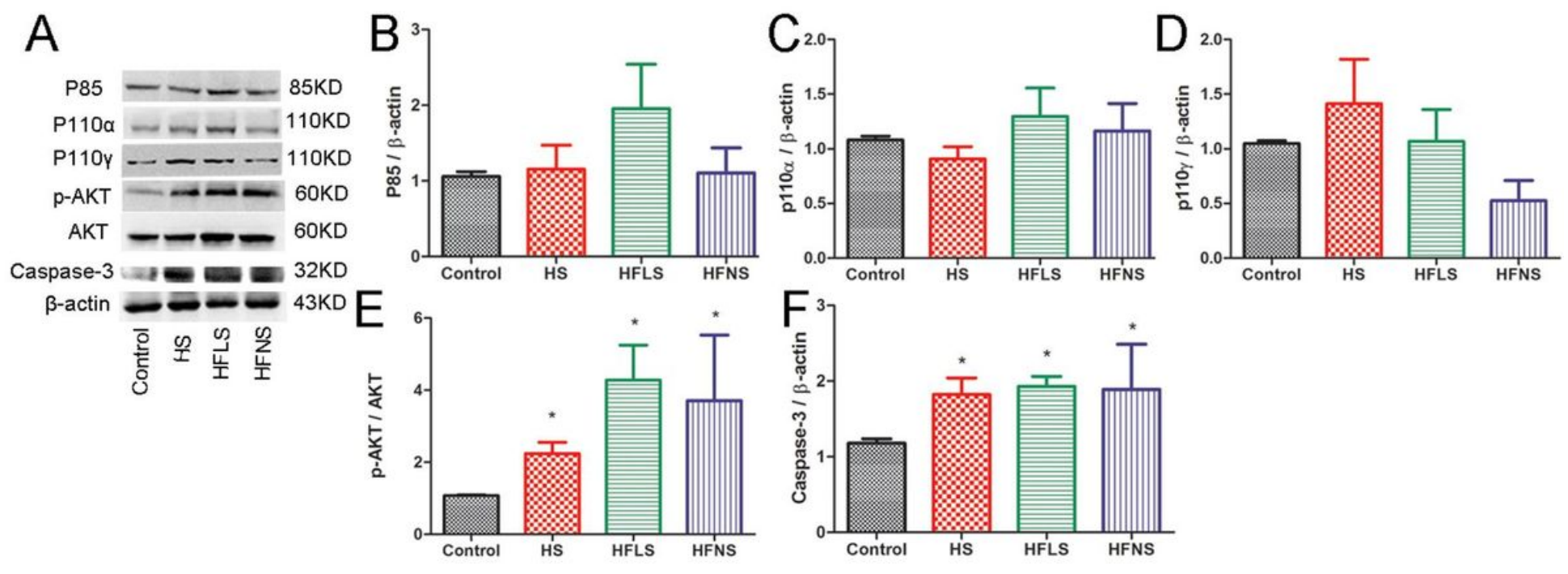

\section{Figure 8}

Effects of hypercaloric diets on the protein expression of PI3K/AKT signal pathway in the ovarian tissues in mice on normal (control) or hypercaloric diets. (A) Western blotting gels of P85, P110区, P110y, p-AKT, 
AKT, caspase-3, and $\beta$-actin; (B) P85/ $\beta$-actin; (C) P110区/ $\beta$-actin; (D) P110y/ $\beta$-actin; (E) p-AKT/AKT; and (F) Caspase-3/ $\beta$-actin. $\beta$-actin was used as a loading control. The data are presented as the mean \pm SE from six different mice for each experimental group $(n=6)$. ${ }^{*} p 0.05$ vs Control.

\section{Supplementary Files}

This is a list of supplementary files associated with this preprint. Click to download.

- supplement1.docx 